\title{
Common origin of modified chaotic inflation, nonthermal dark matter, and Dirac neutrino mass
}

\author{
Debasish Borah, ${ }^{1, *}$ Dibyendu Nanda, ${ }^{1, \dagger}$ and Abhijit Kumar Saha ${ }^{2, \$}$ \\ ${ }^{1}$ Department of Physics, Indian Institute of Technology Guwahati, Assam 781039, India \\ ${ }^{2}$ Theoretical Physics Division, Physical Research Laboratory, Ahmedabad 380009, India
}

(Received 6 November 2019; accepted 24 March 2020; published 7 April 2020)

\begin{abstract}
We propose a minimal extension of the standard model of particle physics to accommodate cosmic inflation, dark matter, and light neutrino masses. While the inflationary phase is obtained from a modified chaotic inflation scenario, consistent with the latest cosmology data, the dark matter particle is a fermion singlet that remains out of equilibrium in the early universe. The scalar field that revives the chaotic inflation scenario by suitable modification also assists in generating tiny couplings of dark matter with its mother particle, naturally realizing the nonthermal or freeze-in type dark matter scenario. Interestingly, the same assisting scalar field also helps in realizing tiny Yukawa couplings required to generate the subelectron volt Dirac neutrino mass from neutrino couplings to the standard model-like Higgs field. The minimality as well as providing a unified solution to all three problems keeps the model predictive at experiments spanning out to all frontiers.
\end{abstract}

DOI: 10.1103/PhysRevD.101.075006

\section{INTRODUCTION}

Several experimental evidences from cosmological and astrophysical experiments strongly favor the presence of a large amount of the nonbaryonic form of matter dubbed as dark matter (DM) in the present universe [1]. However, mysteries surrounding the nature and properties of DM are yet to be resolved by the experiments operating at cosmic, intensity, as well as energy frontiers. It is well known that in order to accommodate the DM candidate in particle physics, an extension of the standard model (SM) of particle physics is required. Depending on the strength of the interactions of DM with the SM particles, different types of DM models have been proposed. Among them, the weakly interacting massive particle (WIMP) paradigm has been the most widely studied dark matter scenario for the past few decades. In this framework, a DM candidate typically with electroweak scale mass and interaction rate similar to electroweak interactions can give rise to the correct DM relic abundance [2], a remarkable coincidence often referred to as the WIMP miracle. Now, if such a type of particles whose interactions are of the order of

\footnotetext{
*dborah@iitg.ac.in

dibyendu.nanda@iitg.ac.in

aks@prl.res.in
}

Published by the American Physical Society under the terms of the Creative Commons Attribution 4.0 International license. Further distribution of this work must maintain attribution to the author(s) and the published article's title, journal citation, and DOI. Funded by SCOAP. electroweak interactions really exist, then we should expect their signatures in various DM direct detection experiments where the recoil energies of detector nuclei scattered by DM particles are being measured. However, the null results at direct detection experiments [3-6] have brought the several implementations of the WIMP scenario into tension. This has also resulted in an increased amount of interest in scenarios beyond the thermal WIMP paradigm where the interaction strength of the DM particle with SM particles can be much lower than electroweak interaction; i.e., DM may be more feebly interacting than in the thermal WIMP paradigm. One of the viable alternatives of the WIMP paradigm, which may be a possible reason behind null results at various direct detection experiments, is to consider the nonthermal origin of DM [7]. For a recent review of such a feebly interacting (or freeze-in) massive particle (FIMP) DM, please see [8]. In the FIMP scenario, the DM candidate does not thermalize with the SM particles in the early universe due to its feeble interaction strength, and the initial abundance of DM is assumed to be zero. At some later stage, DM can be produced nonthermally from decay or annihilation of other particles thermally present in the universe.

Apart from the above mentioned DM problem that brings cosmology and particle physics close to each other in the pursuit of finding a solution, another issue faced by the standard hot big bang cosmology is related to the observed isotropy of the cosmic microwave background (CMB), also known as the horizon problem. This cannot be explained in the standard cosmology where the universe remains radiation dominated throughout the early stages. 
To solve this, the presence of a rapid accelerated expansion phase in the early universe, called inflation $[9,10]$, was proposed. Originally proposed to solve the horizon, flatness, and unwanted relic problem in cosmology $[9,11]$, the inflationary paradigm is also supported by the adiabatic and scale invariant perturbations observed in the CMB $[12,13]$. It turns out that one can accommodate such an early inflationary phase within different particle physics models where one or more scalar fields play the role of inflaton. Chaotic inflation [14,15] models were one of the earliest and simplest scenarios that used power law potentials such as $m^{2} \phi^{2}$ with a scalar field $\phi$. It predicts specific values for inflationary parameters such as the spectral index $n_{s} \sim 0.967$, tensor-to-scalar ratio $r \sim 0.133$ for the number of $e$-folds $N_{e}=60$. However, Planck 2018 results [13] strongly disfavor this simple model due to its large prediction of $r$. To continue using such a simple power law type inflationary scenario, one then has to modify the simplest chaotic inflation potential. There exist several proposals in literature where such attempts have been made. For example, radiative corrections to the inflationary potential [16-18], nonminimal coupling of the inflaton with gravity [19-21], or logarithmic mass correction of the inflaton [22,23] could bring down the value of $r$ within the Planck limit as well as keeping $n_{s}$ within the allowed range. Another interesting proposal exists, where the inflation sector is extended by an additional scalar field [24,25]. The mere interaction between the inflaton and the additional scalar field can revive the model successfully by reducing the magnitude of $r$ below the Planck 2018 limit [13]. It is to be noted that the presence of linear or cubic terms of the inflaton in the Lagrangian can destroy the required flatness for having successful inflation in the simple chaotic inflationary scenarios. This can be ensured by imposing one discrete symmetry $Z_{2}$ under which $\phi$ transforms nontrivially. Now, after the end of inflation, reheating of the universe is extremely essential so that the production of radiation and other matter fields can occur. This also sets the initial condition for the standard big bang cosmology. The energy transfer of inflaton to relativistic matter fields can be realized through the nonperturbative preheating process or the perturbative decay of inflaton. In particular, it was shown in [26] that thermalization of the universe through instant preheating could be one simple and elegant scenario for the $Z_{2}$-odd chaotic inflation model. For a recent work on the $Z_{2}$-odd inflaton field where reheating occurs from annihilation instead of decay, please see $[27,28]$.

Although the fundamental origins of inflation and DM could be disconnected to each other, it is very well motivating to study them in a common framework. In fact, there have been proposals where a single field can play the role of inflaton as well as DM [27-51]. Motivated by such common frameworks, here we study a scenario where inflation and nonthermal DM can find a common origin.
However, unlike a similar proposal [44] where the nonthermal DM field acted as inflaton, here we consider a fermionic nonthermal DM which is produced dominantly from the decay of a scalar field that was present in the thermal bath of the early universe. The decay is assisted by another scalar field that plays a nontrivial role in modifying the minimal chaotic inflation scenario as required by the latest cosmology data mentioned earlier. Thus, although the inflaton and DM fields are not the same, yet they are nontrivially connected by another scalar field that assists in successful inflation and DM production. The presence of the DM sector also opens up the possibility of perturbative inflaton decay at the end of inflation.

To make our framework more minimal and predictive, we also attempt to address the origin of light neutrino masses. Nonzero neutrino mass and large leptonic mixing are well established facts by now [1] while their origin remains unknown as the SM cannot explain them. Apart from neutrino oscillation experiments, cosmology experiments such as Planck also constrain the neutrino sector by putting an upper bound on the sum of absolute neutrino masses $\sum\left|m_{i}\right|<0.12 \mathrm{eV}$ [2]. While the nature of light neutrinos, Dirac or Majorana, remains undetermined at oscillation experiments, popular seesaw models such as [52-55], proposed to account for neutrino masses predict Majorana neutrinos. However, experiments looking for neutrinoless double beta decay $(0 \nu \beta \beta)$, a promising signature of Majorana neutrinos, have not yet found any positive results. Though this does not necessarily rule out the Majorana nature, yet it is motivating to study the possibility of light Dirac neutrinos. This has led to several proposals that attempt to generate tiny Dirac neutrino masses in a variety of ways [56-86]. In a recent work [84], a common origin of light Dirac neutrinos and nonthermal DM was proposed where tiny couplings involved in nonthermal DM and Dirac neutrino mass had a common source from higher dimensional operators. Here we extend that idea to incorporate inflation as well, ${ }^{1}$ within a modified chaotic inflation scenario. We extend the SM by three additional scalar fields and a fermion DM field with suitable discrete symmetries in order to keep the unwanted terms away. Since light neutrinos are of Dirac type, three right-handed neutrinos are present by default. While one of the scalars is the inflaton field and one is the mother particle for DM, the third one assists in inflation as well as DM production. We find that correct DM and neutrino phenomenology can successfully be reproduced in the model while the inflationary parameters predicted by the model remain allowed from Planck 2018 data.

This paper is organized as follows. In Sec. II, we present our model and the corresponding particle spectra, including light Dirac neutrinos. In Sec. III, we discuss the details of

\footnotetext{
${ }^{1}$ See [34,87-92] for earlier attempts in linking neutrino and dark matter with inflation.
} 
inflation in our model followed by the details of the nonthermal fermion DM in Sec. IV. We finally conclude in Sec. V.

\section{THE MODEL}

In this section, we discuss our model, its particle content, additional symmetries, and the Lagrangian of the new fields. As mentioned in the Introduction, we extend the SM with two gauge singlet real scalars $(\phi$ and $\chi)$, one gauge singlet complex scalar field $(\eta)$, one vector-like fermion $(\psi)$, and three right neutrinos $\left(\nu_{R}\right)$. All the new fields considered in the model are singlets under the SM gauge symmetry. We impose two discrete symmetries $Z_{4} \times Z_{4}^{\prime}$ in addition to the SM gauge symmetry, in order to achieve the desired terms in the Lagrangian. ${ }^{2}$ The charge assignments of the new fields under the discrete symmetries are shown in Table I. The SM fields other than the leptons have trivial charges +1 under both the discrete symmetries. We also consider an unbroken global lepton number symmetry $U(1)_{L}$ under which SM leptons as well as $\nu_{R}, \psi$ have unit charges. This ensures the absence of Majorana mass terms for neutrinos via higher dimensional operators, leading to a purely Dirac nature of light neutrinos.

The scalar Lagrangian as followed from the charge assignments is provided by

$$
\begin{aligned}
V(\phi, \chi, \eta)= & \frac{1}{2} m^{2} \phi^{2}+\frac{\lambda_{\phi}}{4} \phi^{4}-\frac{c_{1}}{4}\left(\chi^{2}-\frac{v_{\chi}^{2}}{2}\right) \phi^{2}+\frac{\lambda_{\chi}}{4}\left(\chi^{2}-\frac{v_{\chi}^{2}}{2}\right)^{2} \\
& +m_{\eta}^{2}|\eta|^{2}+\lambda_{\eta}|\eta|^{4}+\frac{\lambda_{\phi \eta}}{2} \phi^{2}|\eta|^{2}+\frac{\lambda_{\chi \eta}}{2}|\eta|^{2}\left(\chi^{2}-\frac{v_{\chi}^{2}}{2}\right) \\
& +\lambda_{H}\left(|H|^{2}-\frac{v^{2}}{2}\right)^{2}+\lambda_{H \eta}|\eta|^{2}\left(|H|^{2}-\frac{v^{2}}{2}\right) \\
& +\frac{\lambda_{\chi H}}{2}\left(\chi^{2}-\frac{v_{\chi}^{2}}{2}\right)\left(|H|^{2}-\frac{v^{2}}{2}\right) \\
& +\frac{\lambda_{\phi H}}{2} \phi^{2}\left(|H|^{2}-\frac{v^{2}}{2}\right)+\left(\frac{\lambda_{R}}{2} \phi \eta \eta \chi+\text { H.c. }\right)
\end{aligned}
$$

where we identify the SM Higgs doublet as $H$ and $v, v_{\chi}$ are the vacuum expectation values (VEV) of the neutral component of $H$ and $\chi$, respectively.

The relevant part of the fermionic Lagrangian consistent with the charge assignments of the fields is given by

$$
\mathcal{L}_{F} \supset m_{\psi} \bar{\psi} \psi+\left(\frac{\xi_{\chi \psi} \chi^{2}}{M_{P}}+\frac{\xi_{\phi \psi} \phi^{2}}{M_{P}}+\frac{\xi_{\eta \psi}|\eta|^{2}}{M_{P}}\right) \bar{\psi} \psi
$$

\footnotetext{
${ }^{2}$ To keep our discussion minimal, we have adopted such discrete symmetries. UV completion can be achieved by suitable gauge symmetries, for example, Abelian gauge extensions [93]. A recent work where $B-L$ gauge symmetry leading to light Dirac neutrinos and a residual $Z_{2} \times Z_{2}^{\prime}$ symmetry can be found in [94].
}

TABLE I. Charge assignments of the new fields present in the model under the discrete symmetries.

\begin{tabular}{ccccccc}
\hline \hline & $l_{L}$ & $\nu_{R}$ & $\psi$ & $\chi$ & $\eta$ & $\phi$ \\
\hline$Z_{4}$ & 1 & -1 & 1 & -1 & 1 & -1 \\
$Z_{4}^{\prime}$ & $i$ & $i$ & -1 & 1 & $i$ & -1 \\
\hline \hline
\end{tabular}

$$
+\left(\frac{\xi \eta \chi \bar{\psi} \nu_{R}}{M_{P}}+\text { H.c. }\right)+\left(\frac{y \chi \overline{l_{L}} \tilde{H} \nu_{R}}{M_{P}}+\text { H.c. }\right) .
$$

Here $l_{L}$ denotes the usual lepton doublet of SM and $M_{P}$ is the Planck mass. The bare mass term for vectorlike fermion $\psi$ is the only renormalizable term involving $\psi$ while all other terms arise only at the dimension five level or higher. The discrete symmetries prevent coupling between the SM lepton doublet and right-handed neutrinos at tree level, which would require Dirac neutrino Yukawa to be finetuned at the level of $\mathcal{O}\left(10^{-12}\right)$ or even smaller. The discrete symmetries also help in preventing the Majorana mass term of right-handed neutrinos, which is needed to ensure the pure Dirac nature of light neutrinos. After $\chi, H$ acquire nonzero VEVs, the light neutrino masses arise as

$$
M_{\nu}=y \frac{v v_{\chi}}{2 M_{P}} .
$$

For $v \approx 10^{2} \mathrm{GeV}, v_{\chi} \approx 10^{9} \mathrm{GeV}$, it is possible to generate $M_{\nu} \approx 0.1 \mathrm{eV}$ for Yukawa couplings $y \approx 10^{-2}$. The light neutrino mixing will arise from the structure of $y$ in the flavor basis, which is not restricted by the symmetries of the model, and hence it is possible to fit it with observed mixing [1] without having any consequence for DM and the inflation sector as we discuss below. Some of the higher dimensional terms involving $\psi$ can also generate a new contribution to $\psi$ mass after one or more of the scalar fields acquire nonzero VEV. However, since $\psi$ already has a bare mass term, it is always possible to adjust its mass at a suitable value by adjustment of different relative contributions.

\section{INFLATION}

Here we discuss the dynamics of inflation and its predictions in detail. The inflation is governed by the following potential:

$V(\phi, \chi)=\frac{m^{2} \phi^{2}}{2}-\frac{c_{1}}{4}\left(\chi^{2}-\frac{v_{\chi}^{2}}{2}\right) \phi^{2}+\frac{\lambda_{\chi}}{4}\left(\chi^{2}-\frac{v_{\chi}^{2}}{2}\right)^{2}$,

where we identify $\phi$ as the inflaton and $\chi$ is the assisting field. We also consider the coupling coefficients $c_{1}$ and $\lambda_{\chi}$ to be real and positive. The global minimum of the $\chi$ field is denoted by $v_{\chi}$, which we assume to be much smaller than $M_{P}$. We also ignore higher order terms of $\phi$, e.g., $\phi^{4}$, by considering the associated coupling coefficients negligibly 

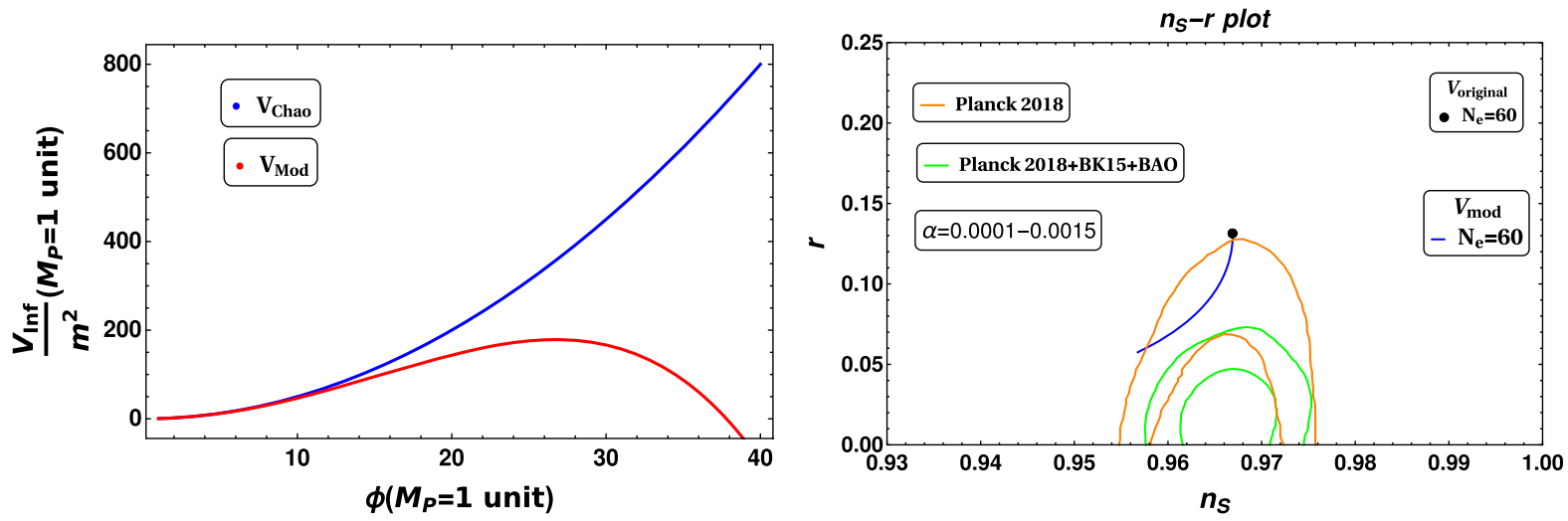

FIG. 1. Left: Sketch of $V_{\text {Inf }}^{\text {eff }}$ given in Eq. (8) for $\alpha=0.0005$ (red curve) and $\alpha=0$ (blue curve). Right: $n_{s}-r$ contour line for $N_{e}=60$ as obtained from our proposed model. For comparison we also show the prediction of the original chaotic inflation model. Furthermore we also include the Planck TT, TE, EE + lowE + lensing and Planck TT, TE, EE + lowE + lensing + BK15 + BAO $1 \sigma$ and $2 \sigma$ allowed contours separately.

small. At the beginning of inflation the $\chi$ field acquires a negative mass squared $\left(\sim c_{1} \phi^{2}\right)$ of order $\mathcal{O}\left(H_{\text {Inf }}^{2}\right)$ where $H_{\text {Inf }}$ is the Hubble parameter during inflation. Thus the $\chi$ field is expected to be driven quickly toward its inflaton field dependent local minimum given by

$$
\left\langle\chi^{2}\right\rangle_{\mathrm{Inf}}=\frac{v_{\chi}^{2}}{2}+\frac{c_{1}}{2 \lambda_{\chi}} \phi^{2} \simeq \frac{c_{1}}{2 \lambda_{\chi}} \phi^{2}
$$

where we assume $v_{\chi}^{2} \ll \frac{c_{1}}{\lambda_{\chi}} \phi^{2}$. Around $\chi=\langle\chi\rangle_{\mathrm{Inf}}$, the effective mass squared of the $\chi$ field is positive and obtained as

$$
\left.m_{\chi}^{2}\right|_{\chi=\langle\chi\rangle_{\mathrm{Inf}}}=c_{1} \phi^{2}+\lambda_{\chi} v_{\chi}^{2} \simeq c_{1} \phi^{2} .
$$

This turns out to be bigger than the $H_{\mathrm{Inf}}^{2} \simeq \frac{m^{2} \phi^{2}}{6 M_{P}^{2}}$ (with suitable choices of $c_{1}$ and $\lambda_{\chi}$ and super-Planckian $\phi$ during inflation), and hence the $\chi$ field is expected to be stabilized at $\langle\chi\rangle_{\text {Inf }}$ with negligible fluctuations [95-98]. Thus inflation occurs along the $\phi$ field direction with the $\chi$ field stabilized at $\langle\chi\rangle_{\text {Inf. }}$. One can obtain the effective inflationary potential by integrating out the heavier field $\chi$ [95-98] [by replacing Eq. (6) into Eq. (5)], which is given by

$$
\begin{aligned}
V_{\mathrm{Inf}}^{\mathrm{eff}} & =\frac{1}{2} m^{2} \phi^{2}-\frac{c_{1}^{2}}{16 \lambda_{\chi}} \phi^{4}, \\
& =\frac{m^{2} \phi^{2}}{2}\left(1-\alpha \phi^{2}\right),
\end{aligned}
$$

where we write $\alpha=\frac{c_{1}^{2}}{8 \lambda_{x} m^{2}}$. From here onwards, for making the analysis simple we shall work with the $M_{P}=1$ unit. The parameter $\alpha$ determines the amount of deformation of the modified chaotic potential from the minimal chaotic inflation scenario. In the left panel of Fig. 1, we plot the effective inflationary potential $V_{\text {Inf }}^{\text {eff }}$ (normalized by $m^{2}$ ) for $\alpha=0$ (blue curve) and $\alpha=0.0007$ (red curve). As we can see, nonzero $\alpha$, associated with the presence of the $\chi$ field, makes the inflationary potential flatter than the minimal one $(\alpha=0)$. The flattening starts to occur near $\phi \sim 10$ in the $M_{P}=1$ unit for $\alpha=0.0007$ while before that the potential merges with the original chaotic inflation potential. Although flattened, the potential becomes unbounded from below at a large value of $\phi$ (e.g., after the maximum at $\phi \sim 30$ for $\alpha=0.0007$ ) as seen from the left panel of Fig. 1. Then in order to have successful inflation we must make an important assumption that inflaton always stays below the maximum of the potential toward the flat part. However, the possibility of tunneling of the inflaton from its minimum to the unstable part of the potential still remains. In that case it is essential to confirm the metastability of the minimum of $\phi$ by calculating the corresponding tunneling probability. We have shown a rough estimate of the decay probability in Appendix A and found it negligibly small.

Before we find the predictions of the model, let us summarize the important conditions or assumptions that we need to ensure in order to realize successful inflation. They are as follow: (i) at the onset of inflation and afterwards the energy of the universe is dominated by the $\phi$ field which implies $\lambda_{\chi} \chi^{4}<\frac{1}{2} m^{2} \phi^{2}$; (ii) the $\chi$ field during inflation is massive compared to the Hubble scale, i.e., $m_{\chi}^{2}>H_{\text {Inf }}^{2}$ so that we can integrate out the $\chi$ field during inflation; (iii) we keep the value of the $\chi$ field sub-Planckian.

Let us proceed to find out the values of the inflationary observables: spectral index $\left(n_{s}\right)$ and tensor to scalar ratio $(r)$ using the modified potential $V_{\text {Inf }}^{\text {eff }}$ in Eq. (8). The analytic expressions for $n_{s}$ and $r$ in our setup are obtained as (in $M_{P}=1$ unit)

$$
\epsilon=\frac{1}{2}\left(\frac{V_{\mathrm{Inf}}^{\prime}}{V_{\mathrm{Inf}}}\right)^{2}=\frac{2}{\phi^{2}}\left[\frac{1-2 \alpha \phi^{2}}{1-\alpha \phi^{2}}\right]^{2},
$$




$$
\eta=\left(\frac{V_{\mathrm{Inf}}^{\prime \prime}}{V_{\mathrm{Inf}}}\right)=\frac{2}{\phi^{2}}\left[\frac{1-6 \alpha \phi^{2}}{1-\alpha \phi^{2}}\right]
$$

where $V^{\prime}=\frac{\partial V}{\partial \phi}$. The number of $e$-foldings can be determined using

$$
N_{e}=\int_{\phi_{\text {end }}}^{\phi^{*}} \frac{\phi\left(1-\alpha \phi^{2}\right)}{2\left(1-2 \alpha \phi^{2}\right)} d \phi,
$$

where $\phi_{\text {end }}$ and $\phi^{*}$ represent the field value at the end of inflation and the point of horizon exit, respectively. The number of $e$-folds is connected to the inflationary parameters through the following relation [99-102]:

$$
N_{e} \simeq 63.3+\frac{1}{4} \ln [\epsilon]+\frac{1}{4} \ln \left[\frac{V_{\mathrm{Inf}}}{\rho_{\mathrm{end}}}\right]+\frac{1}{12} \ln \left[\frac{T_{\mathrm{rh}}^{4}}{\rho_{\mathrm{end}}}\right],
$$

where $V_{\text {Inf }}$ indicates the energy scale of inflation. The energy density at the end of inflation and the reheating temperature of the universe are denoted by $\rho_{\text {end }}$ and $T_{\text {rh }}$, respectively. Once these are known, $N_{e}$ can easily be computed. Now the spectral index and tensor to the scalar ratio in the slow-roll inflation model are defined as

$$
\begin{gathered}
n_{s}=1-6 \epsilon+2 \eta, \\
r=16 \epsilon .
\end{gathered}
$$

The curvature perturbation spectrum is given by

$$
P_{S}=\frac{V_{\mathrm{Inf}}}{24 \pi^{2} \epsilon}=\frac{m^{2} \phi^{4}}{96 \pi^{2}} \frac{\left(1-\alpha \phi^{2}\right)^{3}}{\left(1-2 \alpha \phi^{2}\right)^{2}} .
$$

The observed value of $P_{S}$ is found to be $2.2 \times 10^{-9}$ at a pivot scale $k^{*} \sim 0.05 \mathrm{Mpc}^{-1}$ [2]. Using Eqs. (13) and (14) for different values of $\alpha$ we numerically estimate the magnitudes of $n_{S}$ and $r$ as shown in Table II for the number of $e$-folds $N_{e}=60$. The choice of $N_{e}$ is made following Eq. (12) considering $V_{\text {Inf }}^{1 / 4} \sim 10^{16} \mathrm{GeV}, \rho_{\text {end }}^{1 / 4} \sim 10^{12} \mathrm{GeV}, \epsilon \sim 0.001$, and $T_{\mathrm{rh}} \sim 10^{14} \mathrm{GeV}$ (the estimate of $T_{\mathrm{rh}}$ will be shown shortly in the current section). The corresponding values of $m$ (in $M_{P}=1$ unit) are obtained in Table II using the observed value of $P_{S}$. We also show the predictions of our proposed model in Fig. 1 (right panel) by varying $\alpha$ from 0.0001 to 0.0015. It can be concluded from the right panel of Fig. 1

TABLE II. Predictions for the modified version of the chaotic inflation model in $M_{P}=1$ unit.

\begin{tabular}{lcccc}
\hline \hline No. of $e$-folds & $m$ & $\alpha$ & $n_{s}$ & $r$ \\
\hline$N_{e}=60$ & $5.94 \times 10^{-6}$ & 0.0003 & 0.9661 & 0.1174 \\
& $5.83 \times 10^{-6}$ & 0.0007 & 0.9652 & 0.0970 \\
& $5.59 \times 10^{-6}$ & 0.0011 & 0.9620 & 0.0760 \\
\hline \hline
\end{tabular}

that with the increase of $\alpha$, the value of $r$ can be reduced (in comparison with the minimal form of chaotic inflation) to be consistent with Planck TT, TE, EE + low E + lensing results [13]. However, the model is ruled out if we consider Planck TT, TE, EE+lowE+lensing + BICEP2/KeckArray(BK15) + BAO data [13], which is more stringent than earlier. In addition, unboundedness exists for the effective inflationary potential [Eq. (8)] at a large $\phi$ value, which gives rise to the metastability issue [103]. Below we will see that the addition of a mere higher dimensional term to the inflationary sector will alleviate this problem and in addition make the inflationary predictions consistent with Planck + BK15 + BAO bounds as well.

\section{A. Inflation with higher dimensional operator}

In an effort to make the model consistent with Planck + $\mathrm{BK} 15+\mathrm{BAO}$ data we incorporate a higher dimensional operator (which is perfectly allowed from the charge assignments in Table I) in the inflationary potential [Eq. (5)] given by

$$
V_{\mathrm{HO}}^{\mathrm{Inf}}=\frac{c_{2}}{8 \Lambda^{2}}\left(\chi^{2}-\frac{v_{\chi}^{2}}{2}\right)^{2} \phi^{2}
$$

where we assume $c_{2}$ to be real and positive. We take $\Lambda=M_{P}$ as the natural cutoff scale of the theory. The total inflationary scalar potential is written as $V_{T}^{\mathrm{Inf}}=V(\phi, \chi)+V_{\mathrm{HO}}^{\mathrm{Inf}}$. Similar to the earlier case, here we also integrate out the heavier $\chi$ field which again receives a negative mass-squared larger than the $\mathcal{O}\left(H_{\text {Inf }}^{2}\right)$ at the onset of inflation. Then we obtain the effective inflationary potential which is (in $M_{P}=1$ unit)

$$
V_{\mathrm{Inf}}^{\mathrm{eff}}=\frac{m^{2} \phi^{2}}{2}\left[1-\frac{\beta_{1} \phi^{2}}{16\left(\lambda_{\chi}+\beta_{2} \phi^{2}\right)}\right],
$$

where $\beta_{1}=\frac{2 c_{1}^{2}}{m^{2}}$ and $\beta_{2}=\frac{c_{2}}{2 M_{P}^{2}}=\frac{c_{2}}{2}$ and we also consider $v_{\chi}^{2} \ll \frac{c_{1}}{\lambda_{\chi}}$ (in $M_{P}=1$ unit). Using this effective potential one can calculate the inflationary predictions $n_{s}$ and $r$ using Eqs. (13) and (14) by varying $\beta_{1}$ and $\beta_{2}$ for a fixed value of $\lambda_{\chi} \sim 10^{-8}$. The parameter $m$ would be determined from the observed value of curvature perturbation spectrum $\left(P_{S}\right)$. It is important to note that the inflationary potential in Eq. (17) has no maxima at large values of $\phi$ provided $\beta_{1}<16 \times \beta_{2}$ with $\beta_{1}$ and $\beta_{2}$ being real and positive. This inequality is obtained using the condition $V_{\mathrm{Inf}}^{\mathrm{eff}}>0$ for any arbitrary value of $\phi$. Considering the inequality $\beta_{1}<16 \times \beta_{2}$ is satisfied, the effective inflationary potential [Eq. (17)] due to the presence of higher dimensional operator $V_{\mathrm{HO}}^{\mathrm{Inf}}$ in Eq. (16) is a monotonically increasing function of the $\phi$. Therefore, the inflaton $\phi$ can naturally roll toward the minimum $\phi=0$ from any arbitrary large value. This notable feature of the effective inflationary potential makes the setup more favored unlike 


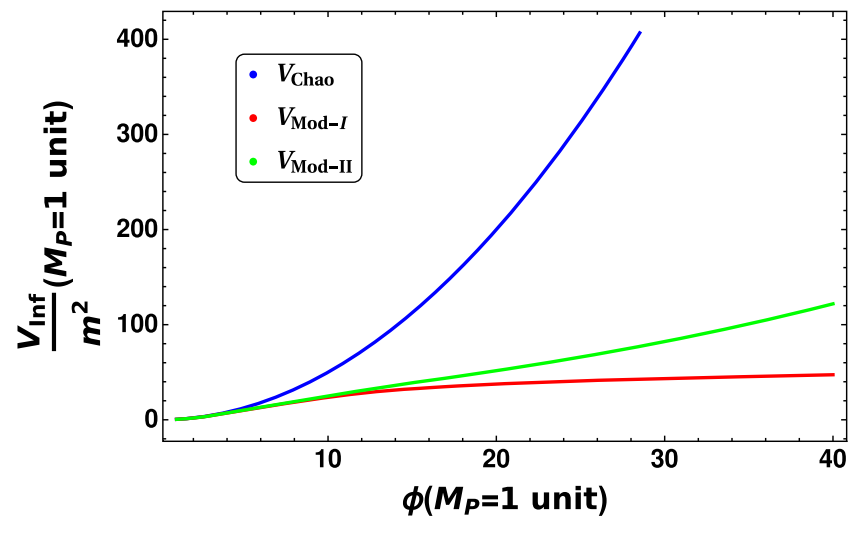

FIG. 2. Sketch of $V_{\text {Inf }}^{\text {eff }}$ in Eq. (17) for reference points I and II of Table III, i.e., $\left\{m, \beta_{1}, \beta_{2}\right\} \rightarrow\left\{6.72 \times 10^{-6}, 1.78 \times 10^{-9}, 1.12 \times\right.$ $\left.10^{-10}\right\}$ (green line) and $\left\{7.65 \times 10^{-6}, 1.78 \times 10^{-9}, 1.25 \times\right.$ $\left.10^{-10}\right\}$ (red line). For comparison, we also include the potential structure of the minimal chaotic potential (blue line).

the previous case $\left(\beta_{2}=0\right)$ where the effective inflationary potential suffers from the issue of unboundedness at large $\phi$.

We have shown a sketch of the effective inflationary potential [Eq. (17)] in Fig. 2 for two benchmark points which exhibit that the effective inflationary potential [Eq. (17)] is much flatter than the one in the minimal chaotic model with only the $m^{2} \phi^{2}$ potential. Here the flattening starts to take place near $\phi \sim 8 M_{P}$. We estimate $N_{e}$ to be 60 corresponding to the inflationary energy scale $\sim 10^{16} \mathrm{GeV}$ and reheat temperature $\sim 10^{14} \mathrm{GeV}$ similar to the earlier case. We also take into account the limit $\beta_{1}<$ $16 \times \beta_{2}$ while scanning the parameter space. In Fig. 3 we show the predictions of the proposed model in the presence of the higher dimensional term [Eq. (16)] in the $n_{s}-r$ plane for $N_{e}=60$. We vary $\beta_{1}$ for a definite $\beta_{2}$ in the right panel while the left panel shows the effect of varying $\beta_{2}$ for a fixed $\beta_{1}$. It is clear that both $\beta_{1}$ and $\beta_{2}$ in Eq. (17) help in
TABLE III. Predictions for the modified version of the chaotic inflation model with a higher order contribution in $M_{P}=1$ unit. We have fixed $\lambda_{\chi}=10^{-8}$.

\begin{tabular}{lccccc}
\hline \hline No. of $e$-folds & $m$ & $\beta_{1}$ & $\beta_{2}$ & $n_{s}$ & $r$ \\
\hline$N_{e}=60$ & $6.72 \times 10^{-6}$ & $1.78 \times 10^{-9}$ & $1.12 \times 10^{-10}$ & 0.970 & 0.038 \\
& $7.65 \times 10^{-6}$ & $1.78 \times 10^{-9}$ & $1.25 \times 10^{-10}$ & 0.975 & 0.057 \\
\hline \hline
\end{tabular}

lowering the value of $r$ such that the inflationary predictions (both $n_{s}$ and $r$ ) successfully fall within the stringent Planck $\mathrm{TT}, \mathrm{TE}+$ low $\mathrm{E}+$ lensing $+\mathrm{BK} 15+\mathrm{BAO}$ bounds. We tabulate two reference points for $N_{e}=60$ in Table III that show the numerical estimates of the inflationary predictions following the redefined potential in Eq. (17).

Now considering $c_{1}=\sqrt{\frac{\beta_{1}}{2}} m \sim 1.11 \times 10^{-11}$ and $\lambda_{\chi} \sim$ $10^{-8}$ as in benchmark point I of Table III and $\phi \sim 15 M_{P}$ during inflation, we find $v_{\chi} \ll 0.5 M_{P}$ in order to maintain the validity of our approximation in Eqs. (6) and (17). In addition, if we consider the mass of the extra scalar field $\left(m_{\chi}\right)$ to be larger than $\mathcal{O}(10) \mathrm{TeV}$, a lower bound on $v_{\chi}$ can also be obtained, which is $v_{\chi} \gtrsim 10^{8} \mathrm{GeV}$ for $\lambda_{\chi} \sim 10^{-8}$. The upper bound on $v_{\chi}$ can be further improved by taking into account the high scale validity of the proposed model. A stronger upper bound $v_{\chi} \lesssim 10^{14} \mathrm{GeV}$ can be found for $\lambda_{\chi} \sim$ $10^{-8}$ if we consider the high scale stability of electroweak vacuum during and after inflation [25]. Moreover, the high scale validity of the model also constrains the magnitude of $\lambda_{\chi H}$ which should be of the same order as $\lambda_{\chi}$ so that it does not alter the renormalisation group (RG) running of $\lambda_{\chi}$ to a great extent. Another important point to note in the proposed setup is the order of magnitudes of $\lambda_{\phi H}$ and $\lambda_{\phi \eta}$, the coupling coefficients of inflaton with SM Higgs and $\eta$, respectively, in Eq. (1). Following [104], it can be shown that $\lambda_{\phi H}, \lambda_{\phi \eta}>\mathcal{O}\left(10^{-6}\right)$ can spoil the required
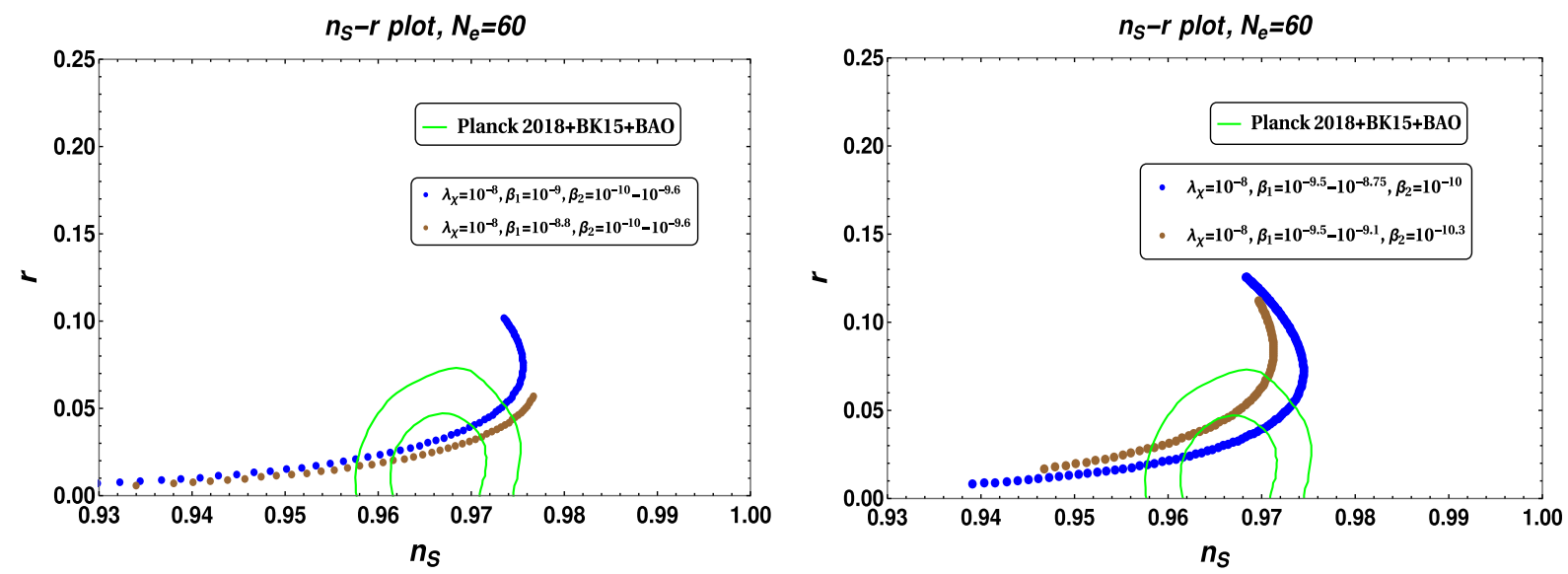

FIG. 3. $n_{s}-r$ contour lines for $N_{e}=60$ as obtained from the redefined inflationary potential of Eq. (17). In the left panel we fix $\beta_{1}$ and vary $\beta_{2}$ while the right panel shows the reverse case. The value of $\lambda_{\chi}$ is kept fixed at $10^{-8}$. We also include Planck TT, TE, EE + lowE + lensing + BK15 + BAO $1 \sigma$ and $2 \sigma$ allowed contours. 
flatness of the chaotic inflationary potential by inducing large radiative corrections. Therefore we keep both $\lambda_{\phi H}$ and $\lambda_{\phi \eta}$ smaller than $\mathcal{O}\left(10^{-6}\right)$ in our scenario.

\section{B. Reheating}

Once inflation ends, the $\phi$ field rolls down and oscillates about its minimum $\phi=0$. Then the reheating process starts to take place. In our model we have various possibilities of energy transfer of inflaton to the relativistic degrees of freedom through $\phi^{2}|H|^{2}, \phi^{2} \chi^{2}, \phi^{2}|\eta|^{2}$ interaction terms. If we consider $\lambda_{\phi H} \gg \lambda_{\phi \chi}, \lambda_{\phi \eta}$, the production of $H$ fields at zero crossing of the $\phi$ field during its oscillation will occur dominantly. Now the $H$ field can decay to SM gauge bosons and fermions with the coupling strength determined by the SM gauge coupling constant and the Yukawa couplings, respectively. Hence it is possible that the produced $H$ field from $\phi$ may decay to SM fields before the oscillating field $\phi$ returns to the minimum of the potential from its maximum value during oscillation. This event is known as instant preheating [26,105-108]. In the process, the effective mass of the $H$ field grows as $m_{H}^{2}=\lambda_{\phi H} \phi^{2}$ when the field $\phi$ rolls up from the minimum of the effective potential. Then the effectively heavy Higgs field decays to SM fields at the moment when it has the greatest mass, i.e., when $\phi$ reaches its maximal value. The number density of produced $H$ fields at the zero crossing of inflaton can be obtained as $[26,105]$

$$
n_{H} \simeq \frac{\lambda_{\phi H}^{3 / 4} \dot{\phi}_{0}^{3 / 2}}{8 \pi^{3}},
$$

where $\dot{\phi}_{0}$ is the velocity of the inflaton around $\phi=0$. For the chaotic inflation model with a quadratic potential, the amplitude of the first oscillation is $\sim 0.1 M_{P}$. Hence the effective mass of produced Higgs field will be around $0.1 \sqrt{\lambda_{\phi H}} M_{P}$, which implies Higgs will be nonrelativistic at that moment. Then the total energy density of the Higgs field can be approximated as [26]

$$
\rho_{H}=m_{H} n_{H} \sim 10^{-14} \lambda_{\phi h}^{5 / 4} M_{P}^{4} .
$$

Now the created $H$ fields will decay completely at the moment $\phi$ reaches its maximum during oscillation if the condition $\left(\Gamma_{H}\right)^{-1} \sim \Delta t$ is obeyed, where $\Delta t$ is the required time for $\phi$ to reach its maximal value during the oscillation. Suppose the decay width of $H$ to SM particles is provided by

$$
\Gamma_{H}=\frac{\delta^{2} m_{H}}{8 \pi},
$$

where $\delta$ is the coupling of Higgs with SM particles. In [26], it is shown that for $\delta^{2} \lambda_{\phi H}^{1 / 2} \sim 5 \times 10^{-4}$, indeed the decay products of $H$ can dominate the energy density of the universe. This can indeed happen if $\lambda_{\phi H} \gtrsim 10^{-8}$, which holds in our model. It is to be noted that the energy dilution of the $\phi$ field can also happen through the perturbative decay $\phi \rightarrow \chi \eta \eta$ as followed from Eq. (1) with the corresponding decay rate

$$
\begin{aligned}
\Gamma_{\phi \rightarrow \chi \eta \eta} \simeq & \frac{\pi \lambda_{R}^{2}}{2 m}\left[\frac{m}{8} \sqrt{m^{2}-4 m_{\chi}^{2}}\right. \\
& \left.-\frac{m_{\chi}^{2}}{2} \log \left\{\frac{1}{2 m_{\chi}}\left(m+\sqrt{m^{2}-4 m_{\chi}^{2}}\right)\right\}\right] .
\end{aligned}
$$

Considering the instant preheating to be the dominant process to transfer the energy of inflaton to relativistic particles, we can try to find a numerical estimate of the reheat temperature. After several oscillations of the inflaton, the complete transfer of the inflaton energy via $\phi \rightarrow$ $H \rightarrow f \bar{f}$ occurs. Then we can use the relation $\rho_{T}=\frac{\pi^{2}}{30} g_{*} T^{4}$ where $g_{*}$ is the number of relativistic degrees of freedom in the thermal bath. At this moment we can assign a reheat temperature to the universe as

$$
T_{\mathrm{rh}}=\left(\frac{30}{\pi^{2} g_{*}}\right)^{1 / 4} \rho_{0}^{1 / 4} \simeq 10^{14} \mathrm{GeV}
$$

where we take $\rho_{0} \simeq \frac{1}{2} m^{2} \phi_{0}^{2}$ with $\phi_{0}$ as the initial amplitude of the $\phi$ oscillation.

\section{DARK MATTER}

Here we present the detailed analysis of DM phenomenology in our model. As pointed out earlier, we consider the fermion singlet field $\psi$ as the DM candidate. From the Lagrangian of the model involving $\psi$ up to the dimension five level, it is clear that $\psi$ does not have any decay mode and hence is stable. This is also ensured by the $Z_{4}^{\prime}$ symmetry that remains unbroken even after the $\chi$ field acquires nonzero VEV. ${ }^{3}$ Thus, the contribution to DM is expected to come entirely from $\psi$ as long as it is the lightest particle charged under the unbroken $Z_{4}^{\prime}$ symmetry. Since $\psi$ is a gauge singlet and it does not have any renormalizable interactions with other particles of the model, it is natural that its interactions with the visible sector particles will remain out of thermal equilibrium in the early universe, a requirement to realize the freeze-in DM scenario. Before the scalar fields acquire VEV, the production of DM can occur from $2 \rightarrow 2$ scattering processes. However, the effective vertex of such scattering diagrams are Planck scale suppressed. This leads to a small ultraviolet (UV) freeze-in contribution to DM abundance [109]. After the

\footnotetext{
${ }^{3}$ It is expected that the $\chi$ field having nonzero VEV will mix with the SM Higgs doublet. However, considering the largeness of $v_{\chi} \gtrsim 10^{9} \mathrm{GeV}$ and small $\lambda_{\chi H} \sim \lambda_{\chi} \simeq 10^{-9}$, the mixing angle turns out to be too small for any phenomenological consequences.
} 
scalar field $\chi$ acquires nonzero VEV, there can be effective two body decay contributions to DM production (infrared freeze-in). This can arise from $\chi \rightarrow \psi \psi \psi$ and $\eta \rightarrow \psi \psi \psi$ with the effective coupling governed by the ratio $v_{\chi} / M_{P}$, which can be as small as $10^{-10}$ for $v_{\chi} \approx 10^{9} \mathrm{GeV}$. Such couplings are quite generic in FIMP scenarios where DM is produced from such two body decays of mother particles [7]. Out of these two contributions, the process $\eta \rightarrow \psi \psi \psi$ will be dominant as $\chi$ has other decay modes whose couplings are not Planck scale suppressed. For example, the $\chi$ field can decay dominantly into a pair of Higgs or a pair of $\eta$. These tree level decays make the field $\chi$ to lose its abundance very quickly. On the other hand, the $\eta$ field gets produced in the thermal bath in the early universe and eventually can freeze-out from the thermal plasma at a later stage. This is similar to the superWIMP scenario [110] where a metastable WIMP decays into a super-weakly interacting dark matter at late epochs. This can happen because $\eta$ can decay only into $\psi$ and the corresponding coupling is very small, thereby allowing the freeze-out to occur earlier. Thus, $\eta$ can decay to $\psi$ while it is in equilibrium and also after its thermal freeze-out. The decay width of mother particle $\eta$,

$$
\Gamma_{\eta \rightarrow \bar{\psi} \nu_{R}}=\frac{y_{\mathrm{eff}}^{2}\left(m_{\eta}{ }^{2}-m_{\psi}^{2}\right)\left(1-\frac{m_{\psi}^{2}}{m_{\eta}{ }^{2}}\right)}{8 \pi m_{\eta}},
$$

where $y_{\text {eff }}=\frac{\xi v_{\chi}}{M_{P}}$ is the effective coupling of the $\eta \psi \nu_{R}$ vertex.

The relic abundance of both $\eta$ and $\psi$ can be found solving the following set of Boltzmann equations which can be expressed as

$$
\begin{gathered}
\frac{d Y_{\eta}}{d x}=-\frac{4 \pi^{2}}{45} \frac{M_{P} m_{\eta}}{1.66} \frac{\sqrt{g_{\star}(x)}}{x^{2}}\langle\sigma v\rangle_{\eta}^{T}\left(Y_{\eta}^{2}-Y_{\eta}^{\mathrm{eq}^{2}}\right) \\
-\frac{M_{P}}{1.66} \frac{x \sqrt{g_{\star}(x)}}{m_{\eta}^{2} g_{s}(x)} \Gamma_{\eta \rightarrow \bar{\psi} \nu_{R}} Y_{\eta}, \\
\frac{d Y_{\psi}}{d x}=\frac{M_{P}}{1.66} \frac{x \sqrt{g_{\star}(x)}}{m_{\eta}^{2} g_{s}(x)} \Gamma_{\eta \rightarrow \bar{\psi} \nu_{R}} Y_{\eta},
\end{gathered}
$$

where the comoving equilibrium number density of $\eta$ is given by

$$
Y_{\eta}^{\mathrm{eq}}=0.145 \frac{g}{g_{s}(T)}\left(\frac{m_{\eta}}{T}\right)^{3 / 2} e^{-\frac{m_{\eta}}{T}},
$$

with $T$ being the temperature of the thermal bath, and $g$ is the internal degrees of freedom of $\eta$. Equation (24) corresponds to the evolution of the comoving number density $\left(Y=\frac{n}{s}\right)$ of mother particle $(\eta)$ as a function of $x=\left(\frac{m_{\eta}}{T}\right)$ where the first term on the right-hand side shows the contribution from the thermal bath to the $Y_{\eta}$, whereas the

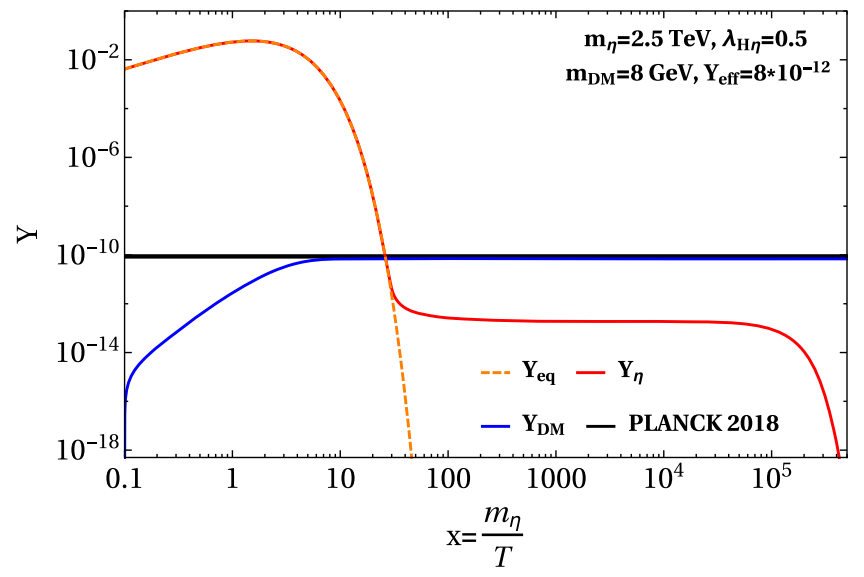

FIG. 4. Variation of comoving number density $Y_{\mathrm{DM}}$ (same as $\left.Y_{\psi}\right)$ as a function of $x\left(=\frac{m_{\eta}}{T}\right)$ for benchmark values of the other parameters. The black solid line stands for the required $Y$ to produce an experimentally observed relic abundance of DM [2] for the chosen set of parameters.

second term stands for the dilution due to the decay of $\eta$ to the DM particles. Similarly, Eq. (25) represents the evolution of the DM particles in the universe from the nonthermal contribution coming from the decay of $\eta$. The effective relativistic degrees of freedom during thermal equilibrium and entropy degrees of freedom are denoted by the usual notations $g_{*}$ and $g_{s}$, respectively, in Eqs. (24) and (25). The $\langle\sigma v\rangle_{\eta}^{T}$ in Eq. (24) stands for the thermally averaged total annihilation cross section of the $\eta$ field as provided in Eq. (B5) of Appendix II. To estimate the relic abundance of DM, we will numerically solve the coupled differential equations (24) and (25) and use the following expression:

$\Omega_{\mathrm{DM}} h^{2}=\frac{h^{2} m_{\mathrm{DM}} s_{0}}{\rho_{\text {crit }}} Y_{\psi}^{(x=\infty)}=2.755 \times 10^{8} \times\left(\frac{m_{\mathrm{DM}}}{\mathrm{GeV}}\right) Y_{\psi}^{(x=\infty)}$,

where $s_{0}, Y_{\psi}^{(x=\infty)}$ are the present entropy density and comoving number density, respectively, and $\rho_{\text {crit }}$ is the critical energy density of the universe. Also, here we identify $m_{\psi}$ as $m_{\mathrm{DM}}$. The parameter $h$ is defined as follows: $h=($ Hubble parameter $) /\left(100 \mathrm{~km} \mathrm{~s}^{-1} \mathrm{Mpc}^{-1}\right)$.

Before performing the numerical analysis we note the parameters that serve important roles in determining the total relic abundance of DM:

$$
\left\{m_{\mathrm{DM}}, m_{\eta}, y_{\mathrm{eff}}, \lambda_{H \eta}\right\} .
$$

In Fig. 4 we have shown the variation of the comoving number densities for both the fields $\eta$ and $\psi$ as a function of $x\left(=\frac{m_{\eta}}{T}\right)$ for a specific choice of values of the relevant parameters as mentioned in the figure. The evolution of $Y_{\eta}$ (red line) clearly shows that $\eta$ was in a thermal bath in the 


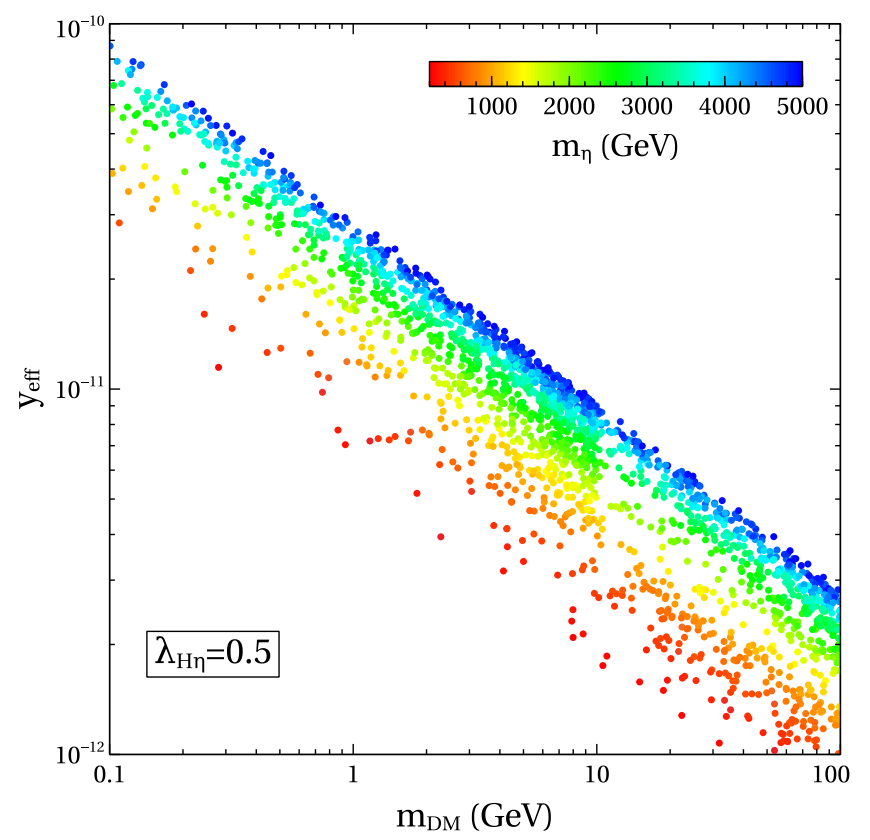

FIG. 5. Allowed parameter space in the $m_{\mathrm{DM}}-y_{\mathrm{eff}}$ plane where the variation of $m_{\eta}$ is shown through color coding.

early universe and went out of equilibrium through the usual freeze-out mechanism and at some later stage continues to decay to DM particle $\psi$. The blue line in Fig. 4 exhibits the evolution of $Y_{\psi}$, which shows that initially the $\psi$ abundance was very small and increases gradually from the decay of the mother particle $\eta$. Figure 5 shows the relic density allowed parameter space in the $m_{\mathrm{DM}^{-}} y_{\text {eff }}$ plane and the colored bar represents the variation of $m_{\eta}$. Here we have varied the DM mass from $100 \mathrm{MeV}$ to $100 \mathrm{GeV}, m_{\eta}$ from $500 \mathrm{GeV}$ to $5 \mathrm{TeV}$, and $y_{\text {eff }}$ from $10^{-12}$ to $10^{-8}$. It can be viewed that for smaller values of $m_{\mathrm{DM}}$ we need larger $y_{\text {eff }}$ considering a fixed value of $m_{\eta}$. As the mass of DM increases, the required number density of DM, i.e., $Y_{\psi}$ (proportional to $\Gamma_{\eta}$ ), has to be smaller to satisfy the correct relic abundance [see Eq. (27)], and hence the decrease in $y_{\text {eff }}$ is observed from Fig. 5. Also, it is seen from Fig. 5 that the increase in $m_{\eta}$ raises $y_{\text {eff }}$ in order to have a correct amount of relic density. This can be understood by looking at Eq. (25), where larger $m_{\eta}$ causes suppression in the value of $Y_{\psi}$, and hence it requires comparatively large $y_{\text {eff }}$.

\section{CONCLUSION}

We have proposed a unified framework to account for nonthermal dark matter, modified chaotic inflation, and sub-electron volt Dirac neutrino mass by minimally extending the standard model. The minimal chaotic inflation scenario, being ruled out by Planck data, is modified by an additional scalar field to bring the predictions for inflationary parameters within the allowed range. The same additional scalar field also assists in generating required tiny couplings for nonthermal DM as well as Dirac neutrino mass by virtue of Planck scale suppressed dimension five operators. We find that for suitable VEV of the additional assisting field of the order $10^{9} \mathrm{GeV}$, it is natural to generate Dirac neutrino Yukawa of the order $10^{-12}-10^{-10}$, which can then generate sub-electron volt Dirac neutrino mass by virtue of neutrino coupling to the SM Higgs. Similar couplings generated for DM coupling to its mother particle also make the realization of the FIMP dark matter scenario natural. After showing the validity of the modified chaotic inflation scenario for suitable benchmark choices of parameters, we numerically find the parameter space that can generate the correct FIMP DM abundance by scanning over the DM mass, mother particle mass, as well as their couplings. Future cosmology data should be able to make this model go through further scrutiny, especially in terms of the inflationary observables. Also the model can be falsified by the observation of neutrinoless double beta decay, which will rule out the pure Dirac nature of light neutrinos, as proposed in this model.

\section{ACKNOWLEDGMENTS}

D. B. acknowledges the support from Indian Institute of Technology Guwahati start-up grant (No. xPHYSUGIITG01152xxDB001), Early Career Research Award from Science and Engineering Research Board (SERB), Department of Science and Technology (DST), Government of India (No. ECR/2017/001873), and Associateship Programme of Inter University Centre for Astronomy and Astrophysics (IUCAA), Pune. D. N. and A. K. S. thank Amit Dutta Banik for some useful discussions while carrying out the work.

\section{APPENDIX A: DECAY PROBABILITY OF FALSE VACUUM}

The decay probability of the false vacuum per unit time and unit volume can be calculated by adopting the semiclassical method popularly known as the bounce solution. A simpler approximate analytic form of the decay probability is given by [111,112]

$$
P_{v} \sim \phi_{I}^{4} e^{-S_{4}}
$$

where $\phi_{I}$ is the starting field value of bounce and $S_{4}$ represents the Euclidean action for the bounce configuration. It can be shown that for a $\lambda \phi^{4}$ type potential $S_{4}$ turns out to be approximately $[113,114]$

$$
S_{4} \simeq-\frac{8 \pi^{2}}{3 \lambda} .
$$

Now the volume of the past light cone is estimated to be $\sim\left(\frac{e^{140}}{M_{P}}\right)^{4}$. With this, the total probability for nucleation of a bubble in the present Hubble volume is [111,112] 


$$
P_{T}=P_{v} \times\left(\frac{e^{140}}{M_{P}}\right)^{4}
$$

In our case the inflationary potential is dominated by the $\phi^{4}$ term where the unboundedness starts to appear. Therefore it is legitimate to apply this simple method to calculate the tunneling probability. Considering $\phi_{I} \sim 30 M_{P}$ where the unboundedness of the inflaton potential appears, and the quartic coupling coefficient of the $\phi$ field is $\lambda \sim-m^{2} \alpha \simeq-10^{-14}, P_{T}$ comes out to be much smaller than unity.

\section{APPENDIX B: ANNIHILATION CROSS SECTIONS OF $\boldsymbol{\eta}$}

Below we provide the analytic expression of thermally averaged cross sections for all the possible annihilation processes of the $\eta$ field,

$$
\begin{gathered}
\sigma_{\eta \eta \rightarrow f \bar{f}}=\frac{\lambda_{H \eta}^{2} m_{f}^{2}\left\{2\left(s-2 m_{f}^{2}\right)-4 m_{f}^{2}\right\} \sqrt{\frac{s-4 m_{f}^{2}}{s-4 m_{\eta}^{2}}}}{16 \pi s\left\{\Gamma_{h}^{2} m_{h}^{2}+\left(s-m_{h}^{2}\right)^{2}\right\}}, \\
\sigma_{\eta \eta \rightarrow h h}=\frac{1}{16 \pi s} \sqrt{\frac{s-4 m_{h}^{2}}{s-4 m_{\eta}^{2}}} \frac{9 g^{2} \lambda_{H \eta}^{2} m_{h}^{4} v^{2}}{4 m_{W}^{2}\left\{\Gamma_{h}^{2} m_{h}^{2}+\left(s-m_{h}^{2}\right)^{2}\right\}}+\frac{3 g \lambda_{H \eta}^{2} m_{h}^{2} v}{\left.m_{w} \sqrt{\Gamma_{h}^{2} m_{h}^{2}+\left(s-m_{h}^{2}\right)^{2}}+4 \lambda_{H \eta}^{2}\right],} \\
\sigma_{\eta \eta \rightarrow W^{+} W^{-}}=\frac{g^{2} \lambda_{H \eta}^{2} m_{W}^{2} v^{2}\left\{\frac{\left(s-2 m_{W}^{2}\right)^{2}}{4 m_{W}^{4}}+2\right\} \sqrt{\frac{s-4 m_{W}^{2}}{s-4 m_{\eta}^{2}}}}{16 \pi s\left\{\Gamma_{h}^{2} m_{h}^{2}+\left(s-m_{h}^{2}\right)^{2}\right\}}, \\
\sigma_{\eta \eta \rightarrow Z Z}=\frac{1}{16 \pi s} \frac{g^{2} \lambda_{H \eta}^{2} m_{Z}^{2} v^{2}\left\{\frac{\left\{s-2 m_{Z}^{2}\right)^{2}}{4 m_{Z}^{4}}+2\right\} \sqrt{\frac{s-4 m_{z}^{2}}{s-4 m_{\eta}^{2}}}}{\cos ^{2} \theta_{W}\left\{\Gamma_{h}^{2} m_{h}^{2}+\left(s-m_{h}^{2}\right)^{2}\right\}},
\end{gathered}
$$

where we define the following:

$$
\begin{aligned}
& m_{f}: \text { mass of SM fermions, } \quad m_{W}: \text { mass of } W \text { boson, } m_{Z}: \text { mass of } Z \text { boson, } \\
& m_{\eta}: \text { mass of } \eta \text { field }, \quad m_{\mathrm{DM}}: \text { mass of DM, } g: S U(2)_{L} \text { gauge coupling, } \\
& v: \text { vacuum expectation value of the SM Higgs, } \theta_{W}: \text { Weinberg angle, } \\
& \Gamma_{h}: \text { decay width of SM Higgs, } \quad s: \text { center of mass energy. }
\end{aligned}
$$

With all these inputs, the total annihilation cross section $\sigma_{T}$ and the thermal average of the cross section $\langle\sigma v\rangle_{\eta}^{T}$ for $\eta$ can be written as

$$
\begin{gathered}
\sigma_{T}=\sigma_{\eta \eta \rightarrow f \bar{f}}+\sigma_{\eta \eta \rightarrow h h}+\sigma_{\eta \eta \rightarrow W^{+} W^{-}}+\sigma_{\eta \eta \rightarrow Z Z} \\
\langle\sigma v\rangle_{\eta}^{T}=\frac{1}{8 m_{\eta}^{4} T K_{2}^{2}\left(\frac{m_{\eta}}{T}\right)} \int_{4 m_{\eta}^{2}}^{\infty} \sigma_{T}\left(s-4 m_{\eta}^{2}\right) \sqrt{s} K_{1}\left(\frac{\sqrt{s}}{T}\right) d s .
\end{gathered}
$$

[1] M. Tanabashi et al. (Particle Data Group), Phys. Rev. D 98, 030001 (2018).

[2] N. Aghanim et al. (Planck Collaboration), arXiv:1807. 06209.

[3] D. S. Akeribone et al. (LUX Collaboration), Phys. Rev. Lett. 116, 161301 (2016).

[4] E. Aprile et al. (XENON Collaboration), Phys. Rev. Lett. 119, 181301 (2017).
[5] X. Cui et al. (PandaX-II Collaboration), Phys. Rev. Lett. 119, 181302 (2017).

[6] A. Tan et al. (PandaX-II Collaboration), Phys. Rev. Lett. 117, 121303 (2016).

[7] L. J. Hall, K. Jedamzik, J. March-Russell, and S. M. West, J. High Energy Phys. 03 (2010) 080.

[8] N. Bernal, M. Heikinheimo, T. Tenkanen, K. Tuominen, and V. Vaskonen, Int. J. Mod. Phys. A 32, 1730023 (2017). 
[9] A. H. Guth, Phys. Rev. D 23, 347 (1981); Adv. Ser. Astrophys. Cosmol. 3, 139 (1987).

[10] A. A. Starobinsky, Phys. Lett. 91B, 99 (1980).

[11] A. D. Linde, Phys. Lett. 108B, 389 (1982).

[12] E. Komatsu et al. (WMAP Collaboration), Astrophys. J. Suppl. Ser. 192, 18 (2011).

[13] Y. Akrami et al. (Planck Collaboration), arXiv:1807. 06211.

[14] A. D. Linde, Phys. Lett. 129B, 177 (1983).

[15] J. Martin, C. Ringeval, and V. Vennin, Phys. Dark Universe 5-6, 75 (2014).

[16] V. N. Senoguz and Q. Shafi, Phys. Lett. B 668, 6 (2008).

[17] K. Enqvist and M. Karciauskas, J. Cosmol. Astropart. Phys. 02 (2014) 034.

[18] G. Ballesteros and C. Tamarit, J. High Energy Phys. 02 (2016) 153.

[19] C. Pallis and Q. Shafi, J. Cosmol. Astropart. Phys. 03 (2015) 023.

[20] N. Kaewkhao and B. Gumjudpai, Phys. Dark Universe 20, 20 (2018).

[21] T. Tenkanen, J. Cosmol. Astropart. Phys. 12 (2017) 001.

[22] S. Kasuya and M. Taira, Phys. Rev. D 98, 123515 (2018).

[23] P. Van Dong, D. T. Huong, D. A. Camargo, F. S. Queiroz, and J. W. F. Valle, Phys. Rev. D 99, 055040 (2019).

[24] K. Harigaya, M. Ibe, M. Kawasaki, and T. T. Yanagida, Phys. Lett. B 756, 113 (2016).

[25] A. K. Saha and A. Sil, Phys. Lett. B 765, 244 (2017).

[26] G. N. Felder, L. Kofman, and A. D. Linde, Phys. Rev. D 59, 123523 (1999).

[27] S. Choubey and A. Kumar, J. High Energy Phys. 11 (2017) 080 .

[28] D. Borah, P. S. B. Dev, and A. Kumar, Phys. Rev. D 99 , 055012 (2019).

[29] L. Kofman, A. D. Linde, and A. A. Starobinsky, Phys. Rev. Lett. 73, 3195 (1994).

[30] L. Kofman, A. D. Linde, and A. A. Starobinsky, Phys. Rev. D 56, 3258 (1997).

[31] A. R. Liddle and L. A. Urena-Lopez, Phys. Rev. Lett. 97, 161301 (2006).

[32] V. H. Cardenas, Phys. Rev. D 75, 083512 (2007).

[33] G. Panotopoulos, Phys. Rev. D 75, 127301 (2007).

[34] A. R. Liddle, C. Pahud, and L. A. Urena-Lopez, Phys. Rev. D 77, 121301 (2008).

[35] N. Bose and A. S. Majumdar, Phys. Rev. D 80, 103508 (2009).

[36] R. N. Lerner and J. McDonald, Phys. Rev. D 80, 123507 (2009).

[37] N. Okada and Q. Shafi, Phys. Rev. D 84, 043533 (2011).

[38] J. De-Santiago and J. L. Cervantes-Cota, Phys. Rev. D 83, 063502 (2011).

[39] R. N. Lerner and J. McDonald, Phys. Rev. D 83, 123522 (2011).

[40] A. de la Macorra, Astropart. Phys. 35, 478 (2012).

[41] V. V. Khoze, J. High Energy Phys. 11 (2013) 215.

[42] F. Kahlhoefer and J. McDonald, J. Cosmol. Astropart. Phys. 11 (2015) 015.

[43] M. Bastero-Gil, R. Cerezo, and J. G. Rosa, Phys. Rev. D 93, 103531 (2016).

[44] T. Tenkanen, J. High Energy Phys. 09 (2016) 049.

[45] L. Heurtier, J. High Energy Phys. 12 (2017) 072.
[46] D. Hooper, G. Krnjaic, A. J. Long, and S. D. Mcdermott, Phys. Rev. Lett. 122, 091802 (2019).

[47] R. Daido, F. Takahashi, and W. Yin, J. High Energy Phys. 02 (2018) 104.

[48] R. Daido, F. Takahashi, and W. Yin, J. Cosmol. Astropart. Phys. 05 (2017) 044.

[49] J. P. B. Almeida, N. Bernal, J. Rubio, and T. Tenkanen, J. Cosmol. Astropart. Phys. 03 (2019) 012.

[50] A. Torres Manso and J. G. Rosa, J. High Energy Phys. 02 (2019) 020.

[51] S.-M. Choi, Y.-J. Kang, H. M. Lee, and K. Yamashita, J. High Energy Phys. 05 (2019) 060.

[52] P. Minkowski, Phys. Lett. 67B, 421 (1977).

[53] M. Gell-Mann, P. Ramond, and R. Slansky, arXiv:1306. 4669.

[54] R. N. Mohapatra and G. Senjanovic, Phys. Rev. Lett. 44, 912 (1980).

[55] J. Schechter and J. W. F. Valle, Phys. Rev. D 22, 2227 (1980).

[56] K. S. Babu and X. G. He, Mod. Phys. Lett. A 04, 61 (1989).

[57] J. T. Peltoniemi, D. Tommasini, and J. W. F. Valle, Phys. Lett. B 298, 383 (1993).

[58] S. Centelles Chulia, E. Ma, R. Srivastava, and J. W. F. Valle, Phys. Lett. B 767, 209 (2017).

[59] A. Aranda, C. Bonilla, S. Morisi, E. Peinado, and J. W. F. Valle, Phys. Rev. D 89, 033001 (2014).

[60] P. Chen, G.-J. Ding, A. D. Rojas, C. A. Vaquera-Araujo, and J. W. F. Valle, J. High Energy Phys. 01 (2016) 007.

[61] E. Ma, N. Pollard, R. Srivastava, and M. Zakeri, Phys. Lett. B 750, 135 (2015).

[62] M. Reig, J. W. F. Valle, and C. A. Vaquera-Araujo, Phys. Rev. D 94, 033012 (2016).

[63] W. Wang and Z.-L. Han, J. High Energy Phys. 04 (2017) 166.

[64] W. Wang, R. Wang, Z.-L. Han, and J.-Z. Han, Eur. Phys. J. C 77, 889 (2017).

[65] F. Wang, W. Wang, and J. M. Yang, Europhys. Lett. 76, 388 (2006).

[66] S. Gabriel and S. Nandi, Phys. Lett. B 655, 141 (2007).

[67] S. M. Davidson and H. E. Logan, Phys. Rev. D 80, 095008 (2009).

[68] S. M. Davidson and H. E. Logan, Phys. Rev. D 82, 115031 (2010).

[69] C. Bonilla and J. W. F. Valle, Phys. Lett. B 762, 162 (2016).

[70] Y. Farzan and E. Ma, Phys. Rev. D 86, 033007 (2012).

[71] C. Bonilla, E. Ma, E. Peinado, and J. W. F. Valle, Phys. Lett. B 762, 214 (2016).

[72] E. Ma and O. Popov, Phys. Lett. B 764, 142 (2017).

[73] E. Ma and U. Sarkar, Phys. Lett. B 776, 54 (2018).

[74] D. Borah, Phys. Rev. D 94, 075024 (2016).

[75] D. Borah and A. Dasgupta, J. Cosmol. Astropart. Phys. 12 (2016) 034.

[76] D. Borah and A. Dasgupta, J. High Energy Phys. 01 (2017) 072.

[77] D. Borah and A. Dasgupta, J. Cosmol. Astropart. Phys. 06 (2017) 003.

[78] S. Centelles Chulia, R. Srivastava, and J. W. F. Valle, Phys. Lett. B 773, 26 (2017). 
[79] C. Bonilla, J. M. Lamprea, E. Peinado, and J. W. F. Valle, Phys. Lett. B 779, 257 (2018).

[80] N. Memenga, W. Rodejohann, and H. Zhang, Phys. Rev. D 87, 053021 (2013).

[81] S. Centelles Chulia, R. Srivastava, and J. W. F. Valle, Phys. Lett. B 781, 122 (2018).

[82] S. Centelles Chulia, R. Srivastava, and J. W. F. Valle, Phys. Rev. D 98, 035009 (2018).

[83] Z.-L. Han and W. Wang, Eur. Phys. J. C 78, 839 (2018).

[84] D. Borah, B. Karmakar, and D. Nanda, J. Cosmol. Astropart. Phys. 07 (2018) 039.

[85] D. Borah and B. Karmakar, Phys. Lett. B 780, 461 (2018).

[86] D. Borah and B. Karmakar, Phys. Lett. B 789, 59 (2019).

[87] R. Allahverdi, B. Dutta, and A. Mazumdar, Phys. Rev. Lett. 99, 261301 (2007).

[88] A. Mazumdar and S. Morisi, Phys. Rev. D 86, 045031 (2012).

[89] A. Mazumdar and J. Rocher, Phys. Rep. 497, 85 (2011).

[90] K. Kohri, A. Mazumdar, and N. Sahu, Phys. Rev. D 80, 103504 (2009).

[91] J. G. Rodrigues, A. C. O. Santos, J. G. Ferreira, and C. A. de S.Pires, arXiv:1807.02204.

[92] D. Kazanas, R. N. Mohapatra, S. Nasri, and V. L. Teplitz, Phys. Rev. D 70, 033015 (2004).

[93] P. Langacker, Rev. Mod. Phys. 81, 1199 (2009).

[94] D. Nanda and D. Borah, arXiv:1911.04703.

[95] X. Dong, B. Horn, E. Silverstein, and A. Westphal, Phys. Rev. D 84, 026011 (2011).

[96] J. L. Evans, T. Gherghetta, and M. Peloso, Phys. Rev. D 92 , 021303 (2015).

[97] L. McAllister, E. Silverstein, A. Westphal, and T. Wrase, J. High Energy Phys. 09 (2014) 123.
[98] W. Buchmuller, E. Dudas, L. Heurtier, A. Westphal, C. Wieck, and M.W. Winkler, J. High Energy Phys. 04 (2015) 058.

[99] A. R. Liddle and S. M. Leach, Phys. Rev. D 68, 103503 (2003).

[100] J. Martin and C. Ringeval, Phys. Rev. D 82, 023511 (2010).

[101] S. Dodelson and L. Hui, Phys. Rev. Lett. 91, 131301 (2003).

[102] E. W. Kolb and M. S. Turner, Front. Phys. 69, 1 (1990).

[103] G. Ballesteros and C. Tamarit, J. High Energy Phys. 09 (2015) 210.

[104] O. Lebedev and A. Westphal, Phys. Lett. B 719, 415 (2013).

[105] G. N. Felder, L. Kofman, and A. D. Linde, Phys. Rev. D 60, 103505 (1999).

[106] C. Armendariz-Picon, M. Trodden, and E. J. West, J. Cosmol. Astropart. Phys. 04 (2008) 036.

[107] M. Desroche, G. N. Felder, J. M. Kratochvil, and A. D. Linde, Phys. Rev. D 71, 103516 (2005).

[108] R. Allahverdi, A. Ferrantelli, J. Garcia-Bellido, and A. Mazumdar, Phys. Rev. D 83, 123507 (2011).

[109] F. Elahi, C. Kolda, and J. Unwin, J. High Energy Phys. 03 (2015) 048.

[110] J. L. Feng, A. Rajaraman, and F. Takayama, Phys. Rev. D 68, 063504 (2003).

[111] S. R. Coleman, Phys. Rev. D 15, 2929 (1977); 16, 1248(E) (1977).

[112] M. Sher, Phys. Rep. 179, 273 (1989).

[113] K. M. Lee and E. J. Weinberg, Nucl. Phys. B267, 181 (1986).

[114] P. B. Arnold, Phys. Rev. D 40, 613 (1989). 